Ann. Biol. anim. Bioch. Biophys., I969, 9 (4), 45I-462.

\title{
INFLUENCE DES VARIATIONS SAISONNI ÈRES DE LA LUZERNE SUR LA CROISSANCE, LA MORTALITÉ ET L'ÉTABLISSEMENT DE LA MATURITÉ SEXUELLE CHEZ LE CAMPAGNOL DES CHAMPS (MICROTUS ARVALIS)
}

Lise MARTINET et Monique MEUNIER

aver la collaboration technique de J.-M. Lhoste et la collaboration statistique de Aline Solari

Station centrale de Physiologie animale, Centre national de Recherches zootechniques, 78 - Jouy-en-Josas Institut national de la Recherche agronomique

\section{SOMMAIRE}

Les interactions de la végétation et de la durée de la photopériode sur la croissance, la mortalité et l'établissement de la maturité sexuelle ont été étudiées chez le Carnpagnol des Champs.

Les animaux ont été élevés dès leur naissance sous une photopériode claire de to $h$ ou de $15 \mathrm{~h}$ par $24 \mathrm{~h}$ et nourris avec de la luzerne fauchée soit au printemps, soit à l'automne, à deux stades végétatifs différents (30 $\mathrm{cm}$ et préfloraison).

La mortalité au cours du premier mois de la vie est plus élevée, la croissance corporelle, la fertilité des mâles et des femelles plus faible dans les lots nourris avec de la luzerne fauchée à l'automne plutôt qu'au printemps, au stade végétatif de préfloraison, plutôt qu'au stade $30 \mathrm{~cm}$, et soumis à une photopériode claire de $10 \mathrm{~h}$ plutôt qu'à une de $15 \mathrm{~h}$.

Lorsque sont associés les trois facteurs : stade végétatif de la luzerne, cycle de végétation et durée de la photopériode, il est possible d'expliquer en partie les cycles saisonniers observés dans la nature chez les populations de Campagnols.

\section{INTRODUCTION}

Le Campagnol des champs, Microtus arvalis, présente un cycle sexuel relativement régulier (DELOST, I955; BERNARD, I964; MAR'TINET, I967). D'autre part, l'effet favorable des photopériodes claires longues ou croissantes sur le poids des testicules et le nombre de spermatozoïdes chez le mâle, la fréquence de la gestation et la taille des portées chez la femelle, a été bien mis en évidence (LrCCYK, I962; MARTINET I 966 ). 
Cependant, s'il existe chez Microtus arvalis une photopériode claire optimum,

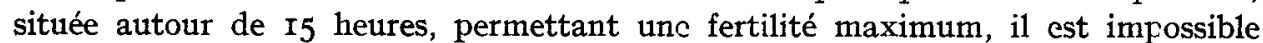
même avec des photofériodes claires aussi courtes que 5 heures par 24 heures, d'empêcher l'apparition de la maturité sexuelle chez le mâle ou la femclle, ou de provơquer des régressions testiculaires complètes chez le mâle adulte.

Il semble donc que la variation de la durée du jour ne soit fas le facteur déterminant dans le déclenchement ou l'arrêt annucl de la reproduction. Il faut alors fenser à un ou plusicurs autres facteurs, agissant ou non cn syrergie avcc le phctopériodisme.

L'étude des populations sauvages de Campaşnols nous a conduit à envisager une certaine relation entre le cycle végétatif des plantes, la luzerne notamment, consommées par les animaux et le cycle sexucl saisonnier. Existc-t-il dans la luzerne des facteurs susceptibles de contrôler la reproduction? Ce sont les expériencos relatives à ce problème qui sont rapportées dans ce travail.

\section{MA'TÉRIEL ET MÉTHODES}

Ce travail a été réalisé à partir de Campagnols élevés sous des conditions contrôlées de lumière et de température et nourris avec de la luzerne récoltée au cours des années 1967 et 1968 .

La luzerne utilisée, provenant d'une luzernière en $2^{\mathrm{e}}$ et $3^{\mathrm{e}}$ année d'exploitation, a été fauchée, congelée immédiatement dans la neige carbonique, puis conservée à $-15^{\circ} \mathrm{C}$ en attendant d'être distribuée. Elle a été prélevée :

- en 1967 :

en avril, au stade $30 \mathrm{~cm}$ du $\mathrm{I}^{\mathrm{er}}$ cycle de végétation = Printemps $\mathrm{I}$, en juillet, au stade $30 \mathrm{~cm}$ du $3^{\mathrm{e}}$ cycle de végétation $=$ Été $\mathrm{I}$, en octobre, au stade $30 \mathrm{~cm}$ du $4^{\mathrm{e}}$ cycle de végétation $=$ Automne $\mathrm{I}$.

- en 1968 :

en avril, au stade $3 \circ \mathrm{cm}$ du I $\mathrm{I}^{\text {er }}$ cycle de végétation $=$ Printemps I, en mai, au stade préfloraison du $\mathrm{I}^{\mathrm{er}}$ cycle de végétation = Printemps II, en septembre, au stade $30 \mathrm{~cm}$ de $4^{\mathrm{e}}$ cycle de végétation $=$ Automne I, en octobre, au stade préfloraison du $4^{\text {e }}$ cycle de végétation $=$ Automne II.

Les expériences sur les animaux ont été conduites en décembre 1967 et décembre 1968 suivant le plan expérimental présenté dans le tableau I ; elles ont permis de comparer le rôle sur la croissance et la fertilité des Campagnols de luzerne soit au même stade de développement, mais provenant de cycles de végétation différents, soit à des stades de développement différents et d'étudier les interactions possibles avec la photopériode.

Les animaux ont été placés dans les conditions expérimentales de lumière et d'alimentation dès leur naissance.

Les portées ont été pesées au sevrage et à trente jours, le nombre de morts enregistré.

Les mâles ont été tués et pesés à 45 jours ; leurs testicules et vésicules séminales pesés, les épididymes broyés suivant la technique de DounCe (I943) pour évaluer les réserves épididymaires, le fructose des vésicules séminales dosé suivant la technique de MaNN (1946).

Les femelles ont été tuées et pesées à 35 jours, après avoir été accouplées pendant 48 heures avec un mâle fertile (en 1967) ou après avoir reçu une injection intrapéritonéale de 1,25 UI d'hormone chorionique gonadotrope (en I 968). Les ovaires ont été prélevés, les corps jaunes et les œufs dénombrés par examen microscopique des coupes sériées des ovaires, du périsac ovarien et des oviductes.

En 1967 , le nombre de femelles des lots soumis à un éclairement quotidien de ro heures étant trop faible, il n'a pas été possible de tenir compte des résultats concernant ce groupe.

Le dépouillement des résultats a été fait à l'aide d'analyses de variance pour les poids du corps et des organes génitaux, à l'aide du test de $\chi^{2}$ pour le nombre de jeunes mourant entre $\circ$ et $3 \circ$ jours ou le nombre de femelles ovulant. 
TABLEAU I

Schéma expérimental auquel les animaux ont été soumis dès leur naissance

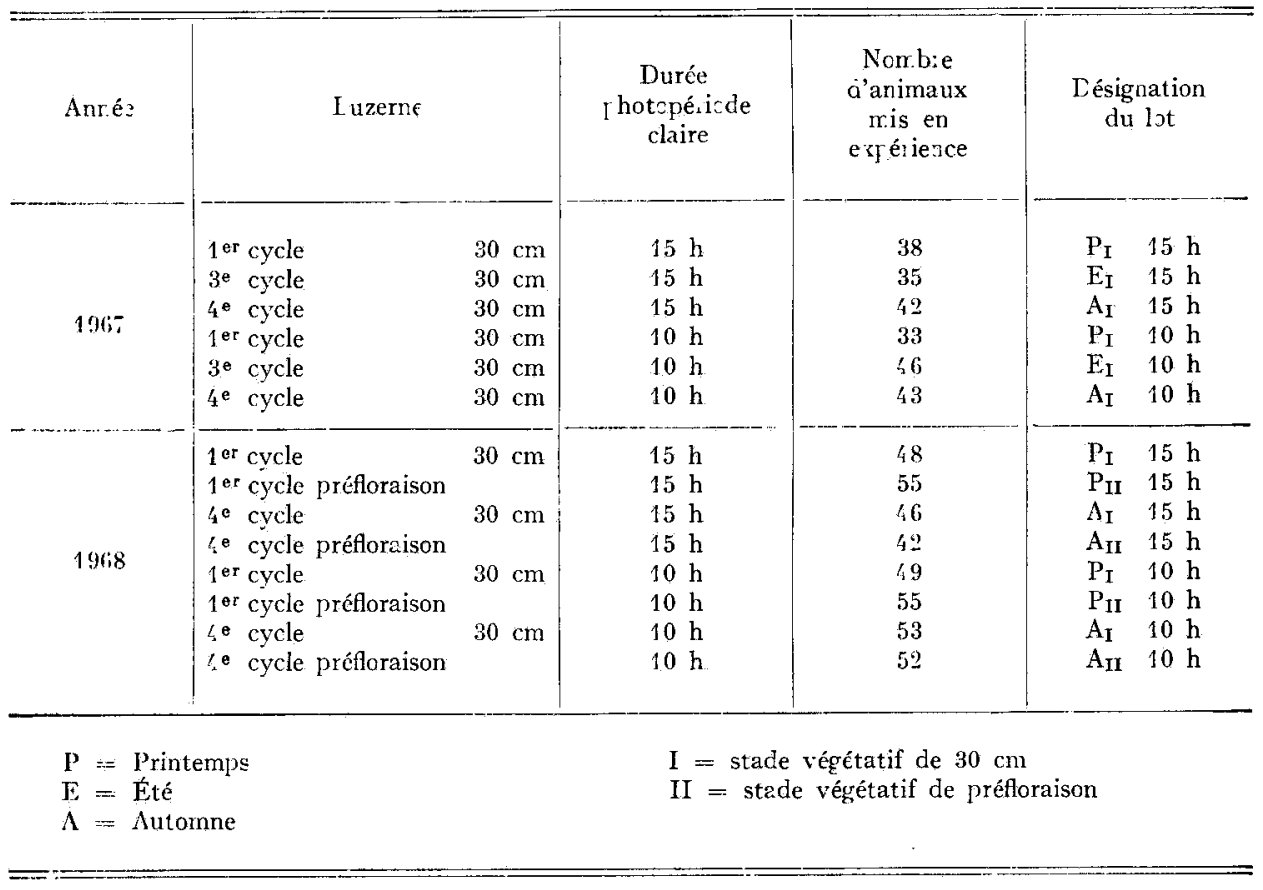

\section{RÉSULTATS}

Trois facteurs et leur interaction possible ont été pris en considération : la saison à laquelle a été fauchée la luzerne, le stade de développement de la luzerne et la durée quotidienne d'éclairement.

\section{Croissance corporelle de la naissance à 30 jours (tab1. 2, fig. I)}

La croissance a été mesurée par le poids des jeunes au sevrage ( 15 j) des femelles à 30 et 35 jours et des mâles à 30 et 45 jours.

Dès le sevrage, une différence dans la croissance corporelle apparaît entre les 8 lots, différence qui s'accentue avec l'âge.

Chez le mâle, les trois facteurs étudiés agissent sur le poids du corps.

En I967, il n'existe pas de différence entre les poids du corps des animaux des 3 lots Printemps, Été, Automne $5_{5}$ heures de lumière ; mais lorsque l'éclairement quotidien est réduit à ro heures, le poids du corps est plus faible dans les lots Été et Automne que dans le lot Printemps.

En I968, les poids du corps sont toujours plus faibles dans les lots recevant de la luzerne d'Automne que dans ceux recevant de la luzerne de Printemps pour un stade végétatif identique. 
La croissance chez des animaux nourris avec de la luzerne au stade préfloraison est toujours plus lente que celle des animaux nourris avec de la luzerne au stade $30 \mathrm{~cm}$.

D'autre part, la comparaison de l'ensemble des résultats, faite année par année grâce à des analyses de variance à deux facteurs et deux niveaux, a permis de montrer que la croissance était toujours significativement plus élevée sous I $_{5}$ heures

TABLEAU 2

Modifications de la croissance corporelle des Campagnols mâles et femelles en fonction de la qualité de la luzerne et de la durée quotidienne d'éclairement

\begin{tabular}{|c|c|c|c|c|c|c|c|}
\hline \multirow{2}{*}{ Année } & \multirow{2}{*}{\multicolumn{2}{|c|}{ Lots }} & \multirow{2}{*}{$\begin{array}{c}\text { Poids } \\
\text { au sevrage } \\
\text { en } \mathrm{g} \pm S_{m}\end{array}$} & \multicolumn{2}{|c|}{ Poids des mâles } & \multicolumn{2}{|c|}{ Poids de femelles } \\
\hline & & & & à $30 \mathrm{j}$ & à $45 \mathrm{j}$ & à $30 \mathrm{j}$ & à $35 \mathrm{j}$ \\
\hline 1967 & $\begin{array}{l}P_{I} \\
E_{I} \\
A_{I} \\
P_{I} \\
E_{I} \\
A_{I}\end{array}$ & $\begin{array}{l}15 \mathrm{~h} \\
15 \mathrm{~h} \\
15 \mathrm{~h} \\
10 \mathrm{~h} \\
10 \mathrm{~h} \\
10 \mathrm{~h}\end{array}$ & $\begin{array}{l}7,5 \pm 0,4 \\
7,7 \pm 0,3 \\
7,5 \pm 0,4 \\
7,3 \pm 0,5 \\
6,8 \pm 0,8 \\
6,7 \pm 0,6\end{array}$ & $\begin{array}{l}15,7 \pm 0,8 \\
17,7 \pm 0,9 \\
16,9 \pm 0,7 \\
16,2 \pm 0,7 \\
16,4 \pm 1,0 \\
15,6 \pm 0,6\end{array}$ & $\begin{array}{l}26,3 \pm 1,4 \\
23,9 \pm 0,9 \\
25,5 \pm 1,1 \\
24,6 \pm 1,1 \\
21,9 \pm 0,6 \\
21,7 \pm 0,7\end{array}$ & $\begin{array}{c}14,8 \pm 0,7 \\
14,0 \pm 0,7 \\
14,1 \pm 0,4 \\
- \\
-\end{array}$ & $\begin{array}{c}16,8 \pm 0,7 \\
15,4 \pm 0,7 \\
16,7 \pm 0,4 \\
\pm \\
- \\
-\end{array}$ \\
\hline 1968 & $\begin{array}{l}P_{I} \\
P_{I I} \\
A_{I} \\
A_{I I} \\
P_{I} \\
P_{I I} \\
A_{I} \\
A_{I I}\end{array}$ & $\begin{array}{ll}15 \mathrm{~h} \\
15 \mathrm{~h} \\
15 \mathrm{~h} \\
15 \mathrm{~h} \\
10 \mathrm{~h} \\
10 \mathrm{~h} \\
10 \mathrm{~h} \\
10 \mathrm{~h}\end{array}$ & $\begin{array}{l}8,2 \pm 0,4 \\
7,0 \pm 0,7 \\
6,9 \pm 0,2 \\
6,2 \pm 0,3 \\
8,7 \pm 0,4 \\
6,3 \pm 0,3 \\
6,8 \pm 0,3 \\
6,4 \pm 0,3\end{array}$ & $\begin{array}{l}21,6 \pm 0,4 \\
15,4 \pm 0,4 \\
15,3 \pm 0,8 \\
15,0 \pm 0,3 \\
17,7 \pm 0,8 \\
13,7 \pm 0,5 \\
15,0 \pm 0,4 \\
13,3 \pm 0,3\end{array}$ & $\begin{array}{l}27,4 \pm 0,7 \\
22,1 \pm 0,6 \\
22,5 \pm 1,0 \\
19,5 \pm 0,5 \\
23,1 \pm 1,3 \\
21,0 \pm 1,3 \\
21,1 \pm 0,6 \\
18,3 \pm 0,5\end{array}$ & $\begin{array}{l}16,1 \pm 0,3 \\
14,3 \pm 0,2 \\
14,3 \pm 0,2 \\
14,4 \pm 0,3 \\
18,2 \pm 0,5 \\
11,8 \pm 0,2 \\
14,8 \pm 0,3 \\
11,6 \pm 0,2\end{array}$ & $\begin{array}{l}18,0 \pm 0,4 \\
15,9 \pm 0,4 \\
16,7 \pm 1,0 \\
16,7 \pm 0,3 \\
21,3 \pm 0,8 \\
15,6 \pm 0,6 \\
17,1 \pm 0,4 \\
14,2 \pm 0,9\end{array}$ \\
\hline
\end{tabular}

$10 \mathrm{~h}$ lumière/24 heures

Variations dues aux facteurs

\begin{tabular}{|c|c|c|c|c|c|c|c|c|c|}
\hline Lot & $\begin{array}{c}\text { Poids } \\
\text { des } \\
\varnothing\end{array}$ & $\begin{array}{c}\text { Varia- } \\
\text { tion } \\
\text { totale }\end{array}$ & $\begin{array}{l}\downarrow \\
\text { Stade }\end{array}$ & $\begin{array}{c}t \\
\text { Saison }\end{array}$ & Lumière & $\begin{array}{c}\downarrow \\
\text { Saison }\end{array}$ & $\begin{array}{l}\text { Stade } \\
\downarrow\end{array}$ & $\begin{array}{l}\text { Varia- } \\
\text { tion } \\
\text { totale }\end{array}$ & $\begin{array}{c}\text { Poids } \\
\text { des } \\
\delta\end{array}$ \\
\hline$P_{I}$ & 27,4 & & & & oس & & 9 & & 23,1 \\
\hline$P_{I I}$ & 22,1 & & & & $0 \ldots 0$ & & & & 21,0 \\
\hline $\mathbf{A}_{\mathbf{I}}$ & 22,5 & & & & $0 \cdots 0$ & & & & 21,1 \\
\hline $\mathbf{A}_{\text {II }}$ & 19,5 & & 8 & & $0 \cdots \infty$ & & & & 18,3 \\
\hline & q & & & & & & & & q \\
\hline $\mathbf{P}_{\mathbf{I}}$ & 18,0 & & & & $0-0$ & & & & 21,3 \\
\hline $\mathbf{P}_{\text {II }}$ & 15,9 & & & & $0 \ldots 0$ & & & & 15,6 \\
\hline $\mathbf{A}_{\mathbf{I}}$ & 16,7 & & & & $0 \cdots 0$ & & & & 17,1 \\
\hline $\mathbf{A}_{\text {II }}$ & 16,7 & & & & $0-0$ & & & & 14,2 \\
\hline
\end{tabular}

FIG. I. - Analyse de la variation du poids du corps des mâles et des femelles, en fonction des trois facteurs: stade de développement de la luzerne, saison de la fenaison et durée de la photopériode

$$
\begin{array}{ll}
\ldots \ldots \ldots \ldots & \begin{array}{l}
\text { Non significatif } \\
\text { Significatif }
\end{array} \\
===== & \text { Hautement significatif }
\end{array}
$$


que sous Io heures de lumière par 24 heures et qu'il n'existait pas d'interaction entre la lumière et, soit l'effet saison de la luzerne, soit l'effet stade de développement (tabl. 5).

Chez les femelles. Bien que les résultats semblent identiques, les conclusions sont plus difficiles à tirer ; en effet, l'analyse statistique montre une interaction hautement significative des effets dus à la luzerne et à la durée de la photopériode claire.

Cependant, on peut noter que ce sont les femelles nourries avec de la luzerne de Printemps $30 \mathrm{~cm}$ qui sont les plus grosses et celles nourries avec de la luzerne d'Automne au stade préfloraison et sous to heures de lumière par 24 heures qui sont les plus petites.

2. Mortalité des jeunes de la naissance à 30 jours (tabl. 3, fig. 2)

La mortalité a été calculée à 15 et 30 jours pour l'ensemble des jeunes mis en expérience et à 45 jours en I 968 pour les mâles.

\section{TABLEAU 3}

Modification de la mortalité des Campagnols mâles et femelles de 0 à 30 jours et des mâles de 30 à 45 jours

en fonction de la qualité de la luzerne et de la durée quotidienne d'éclairement

\begin{tabular}{|c|c|c|c|c|c|}
\hline Année & Lots & $\begin{array}{c}\text { Nombre } \\
\text { de jeunes nés }\end{array}$ & $\begin{array}{c}\text { Animaux } \\
\text { morts à } 14 \mathrm{j} \\
(\%)\end{array}$ & $\begin{array}{c}\text { Animaux } \\
\text { morts à } 30 \mathrm{j} \\
(\%)\end{array}$ & $\begin{array}{c}\text { Mâles } \\
\text { morts à } 45 \mathrm{j} \\
(\%)\end{array}$ \\
\hline 1967 & $\begin{array}{lll}\mathrm{P}_{\mathrm{I}} & 15 \mathrm{~h} \\
\mathrm{E}_{\mathrm{I}} & 15 \mathrm{~h} \\
\mathrm{~A}_{\mathrm{I}} & 15 \mathrm{~h} \\
\mathrm{P}_{\mathrm{I}} & 10 \mathrm{~h} \\
\mathrm{E}_{\mathrm{I}} & 10 \mathrm{~h} \\
\mathrm{~A}_{\mathrm{I}} & 10 \mathrm{~h}\end{array}$ & $\begin{array}{l}38 \\
35 \\
42 \\
33 \\
46 \\
43\end{array}$ & $\begin{array}{r}5 \\
11 \\
17 \\
12 \\
4 \\
14\end{array}$ & $\begin{array}{l}11 \\
17 \\
40 \\
12 \\
35 \\
30\end{array}$ & $\begin{array}{l}- \\
\overline{-} \\
=\end{array}$ \\
\hline 1968 & $\begin{array}{ll}\mathrm{P}_{\mathrm{I}} & 15 \mathrm{~h} \\
\mathrm{P}_{\mathrm{II}} & 15 \mathrm{~h} \\
\mathrm{~A}_{\mathrm{I}} & 15 \mathrm{~h} \\
\mathrm{~A}_{I I} & 15 \mathrm{~h} \\
\mathrm{P}_{I} & 10 \mathrm{~h} \\
\mathrm{P}_{\mathrm{II}} & 10 \mathrm{~h} \\
\mathrm{~A}_{\mathrm{I}} & 10 \mathrm{~h} \\
\mathrm{~A}_{\mathrm{II}} & 10 \mathrm{~h}\end{array}$ & $\begin{array}{l}48 \\
55 \\
46 \\
42 \\
49 \\
55 \\
53 \\
52\end{array}$ & $\begin{array}{r}6 \\
29 \\
13 \\
23 \\
23 \\
16 \\
0 \\
50\end{array}$ & $\begin{array}{r}8 \\
26 \\
30 \\
45 \\
39 \\
35 \\
9 \\
64\end{array}$ & $\begin{array}{l}12 \\
43 \\
34 \\
45 \\
42 \\
48 \\
27 \\
64\end{array}$ \\
\hline
\end{tabular}

En 1967 , 1a comparaison du nombre d'animaux vivants à 15 jours et à 30 jours, par rapport au nombre de jeunes nés, montre qu'il existe une différence significative à 30 jours entre les 6 lots. La mortalité est plus importante chez les animaux des lots Eté ro heures et Automne I5 et ro heures que chez ceux des lots Printemps I5 heures et ro heures et Été 15 heures, mais la durée de la photopériodenesemble pas intervenir.

En 1968, on trouve une différence hautement significative, entre l'ensemble des 8 lots, dans les pourcentages d'animaux vivants à 30 jours. La différence du taux de mortalité des animaux soumis à une photopériode claire de 15 heures est due à une augmentation de cette mortalité chez les animaux nourris avec de la luzerne d'Au- 
tomne, ou de la luzerne au stade de la préfloraison. Chez les animaux recevant ro heures de lumière par 24 heures, la très faible mortalité dans le lot Automne I est difficilement explicable; par contre, on remarque que dans le lot Automne II qui réunit les conditions alimentaires et lumineuses de la fin de l'automne dans la nature, la mortalité est maximum.

$15 \mathrm{~h}$ lumière $/ 2 / \mathrm{t}$ heures

$10 \mathrm{~h}$ lumière/2't heures

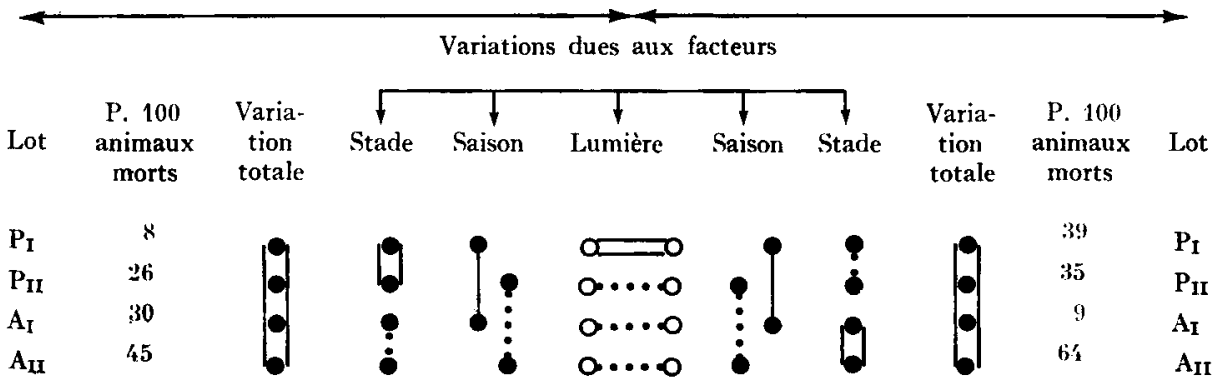

FIG. 2. - Analyse de la variation du nombre d'animaux morts d 30 jours par rapport au nombre d'animaux mis en expérience le jour de leur naissance en fonction des 3 facteurs: stade de développement de la luzerne, saison de la fenaison et durée de la photopériode.

$$
\underline{\ldots \ldots \ldots \ldots} \begin{aligned}
& \text { Non significatif } \\
& =====\begin{array}{l}
\text { Significatif } \\
\text { Hautement }
\end{array} \text { significatif }
\end{aligned}
$$

Entre 30 et 45 jours, la mortalité chez les mâles est pratiquement nulle; on remarque seulement une mortalité assez forte dans le lot Automne I Io heures.

Il semble donc que les facteurs favorisant la mortalité chez les Campagnols au cours du premier mois de la vie soient ceux qui ralentissent la croissance corporelle, à savoir luzerne d'automne, stade végétatif de la préfloraison et photopériode claire de ro heures.

\section{Fertilité chez le mâle (tab1. 4, fig. 3)}

La fertilité a été mesurée par le poids des testicules et des vésicules séminales, le nombre de spermatozoïdes épididymaires et le contenu en fructose des vésicules séminales.

L'analyse des résultats montre que la saison à laquelle est fauchée la luzerne, le stade de développement de cette luzerne et la durée de la photopériode claire ont un effet hautement significatif sur le poids des testicules et des vésicules séminales et qu'il n'y a jamais interaction entre ces trois facteurs (tabl. 5).

La luzerne au stade $30 \mathrm{~cm}$ permet toujours une croissance des testicules et des vésicules séminales supérieure à celle des gonades des animaux recevant de la luzerne au stade de la préfloraison.

De même, la luzerne de Printemps permet toujours une croissance des gonades supérieure à celle de la luzerne d'Automne.

A ces deux effets s'ajoute la stimulation différente des photopériodes claires de I5 heures ou de ro heures.

Le pourcentage de mâles possédant des réserves épididymaires et l'importance de ces réserves varient parallèlement au poids des testicules ; il en est de même pour la teneur en fructose et le poids des vésicules séminales. 


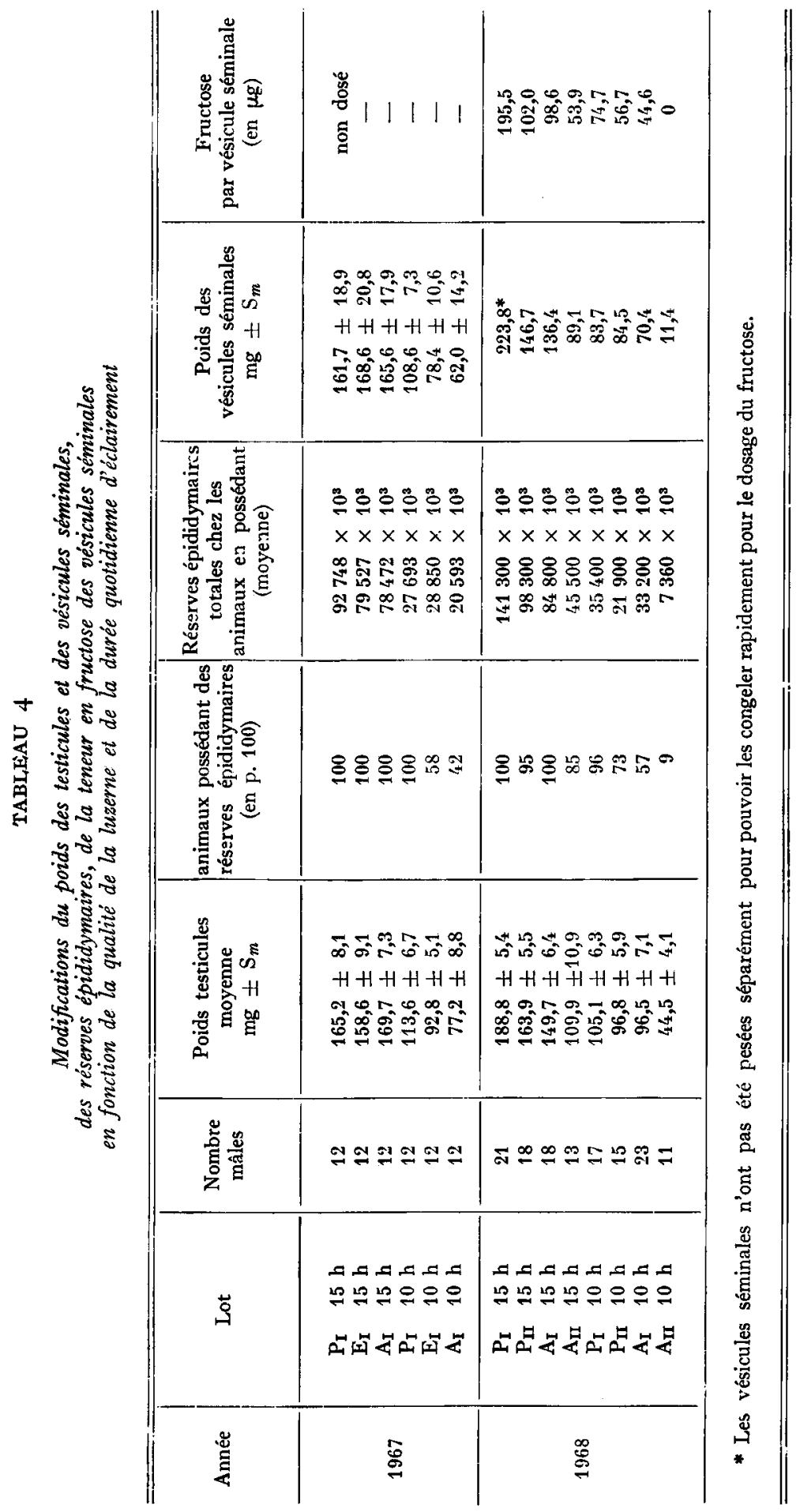


Ainsi, la fertilité est maximum chez les mâles recevant de la luzerne de Printemps au stade $30 \mathrm{~cm}$ sous une photopériode claire de ${ }_{5} 5$ heures. Chez les mâles recevant de la luzerne d'Automne au stade de la préfloraison sous une photopétiode claire de Io heures, l'établissement de la maturité sexuelle semble pratiquement impossible.

$15 \mathrm{~h}$ lumière/2\% heures

10 h lumière/24 heures

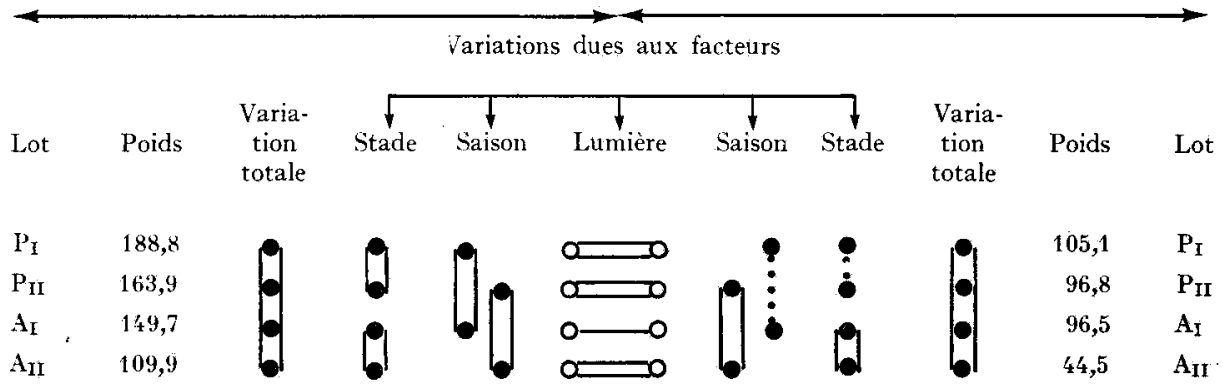

FIG. 3. - Analyse de la variation du poids des testicules en fonction des 3 facteurs: stade de développement de la luzerne, saison de la fenaison et durée de la photopériode

$$
\begin{array}{ll}
\ldots \ldots \ldots \ldots & \begin{array}{l}
\text { Non significatif } \\
\text { Significatif }
\end{array} \\
===== & \text { Hautement significatif }
\end{array}
$$

\section{TABLEAU 5}

Analyse de la variation du poids du corps et des testicules en fonction des facteurs étudiés: stade du développement de la luzerne,

\begin{tabular}{|c|c|c|c|}
\hline Année & Variation due à & $\begin{array}{l}\text { Poids du corps } \\
\text { des mâles }\end{array}$ & Poids des testicules \\
\hline 1967 & $\begin{array}{l}\text { à la saison } \\
\mathbf{P} \text { vs } \mathbf{E} \text { vs } A \ldots \ldots \ldots \ldots \\
\text { à la photopériode } \\
15 \mathrm{~h} \text { vs } 10 \mathrm{~h} \ldots \ldots \ldots \ldots \ldots \\
\text { Interaction } \ldots \ldots \ldots \ldots \ldots \ldots \ldots\end{array}$ & $\begin{array}{l}\text { NS } \\
P<0,01 \\
\text { NS }\end{array}$ & $\begin{array}{c}\text { NS } \\
\mathbf{P}<0,001 \\
\text { NS }\end{array}$ \\
\hline 1968 & $\begin{array}{l}\text { à la saison } \\
P \text { vs } A \ldots \ldots \ldots \ldots \ldots \ldots \ldots \\
\text { au stade végétatif } \\
P_{I} \text { vs } P_{I I} \ldots \ldots \ldots \ldots \ldots \ldots \ldots \\
A_{I} \text { vs } A_{I I} \ldots \ldots \ldots \ldots \ldots \ldots \ldots \\
\text { à la photopériode } \\
15 \text { h vs } 10 h \ldots \ldots \ldots \ldots \ldots \\
\text { Interaction } \ldots \ldots \ldots \ldots \ldots \ldots \ldots\end{array}$ & $\begin{array}{l}\mathrm{P}<0,05 \\
\mathrm{P}<0,01 \\
\mathrm{P}<0,01 \\
\mathrm{P}<0,01 \\
\mathrm{NS}\end{array}$ & $\begin{array}{l}P<0,01 \\
P<0,01 \\
P<0,001 \\
P<0,01 \\
\quad \text { NS }\end{array}$ \\
\hline
\end{tabular}
date de la fenaison et durée de la photopériode

4. Fertilité chez les femelles (tab1. 6, fig. 4)

La fertilité a été mesurée d'une part par le pourcentage de femelles ovulant après accouplement ou injection d'HCG, d'autre part par le nombre d'ovulations par femelle et le pourcentage d'œufs fécondés lorsqu'il y avait eu accouplement. 
Ann. Biol. anim. Bioch. Biophys., I969, 9 (2), 273-280.

\author{
ERRATUM
}

\title{
ÉTUDE ULTRASTRUCTURALE DU SITE DE CONSERVATION DES SPERMATOZOÏDES DANS L'OVAIRE DE POECILIA RETICULATA (POISSON TÉLEOSTÉEN)
}

\author{
B. JALABERT, R. BILLARD
}

avec la collaboration technique de Anne-Marie Escaffre

I,égende de la planche $I$, page 280 :

\section{PLANCHE, I}

Coupe oblique ( $a a^{\prime}$ sur la fig. 2) d'une partie de "cellule hôte " séparée du stroma ovarien (Str) et des capillaires sanguins (Cs) (dont la paroi est riche en vésicules de pinocytose) par la basale (B) où les têtes des spermatozoïdes (S) apparaissent enfoncées dans le cytoplasme et les faisceaux de flagelles $(F)$ emprisonnés dans un réseau lậche de prolongements cytoplasmiques ( $\mathrm{p} \mathrm{Cy}$ ).

Les liaisons entre cellules hôtes sont assurées par des desmosomes (d) et du côté de la lumière du réceptacle séminal $(\mathrm{L})$ les associations entre membranes cellulaires (M) sont du type zona occludens. 


\section{.}


La date de prélèvement, Printemps, Etté ou Automne, n'a pas d'effet sur le pourcentage de femelles ovulant, lorsqu'elles sont soumises à une durée d'éclairement de I5 heures par 24 heures. Par contre, dans les lots où la photopériode claire est de ro heures par 24 heures, il existe une différence significative entre les lots, pour un même stade de développement, en fonction de la date de prélèvement.

\section{TABLEAU 6}

Modifications du nombre de femelles ovulant après accouplement ou injection d'HCG et du nombre de corps jaunes par femelle ayant ovulé en fonction de la qualité de la luzerne et de la durée quotidienne d'éclairement

\begin{tabular}{|c|c|c|c|c|c|}
\hline Année & Lot & $\begin{array}{l}\text { Nombre } \\
\text { femelles }\end{array}$ & $\begin{array}{l}\text { Femelles } \\
\text { ayant ovulé } \\
(\%)\end{array}$ & $\begin{array}{l}\text { Nbre de CJ normaux } \\
\text { chez les femelles } \\
\text { ayant ovulé }\left( \pm S_{m}\right)\end{array}$ & $\begin{array}{l}\text { Eufs fécondés } \\
(\%)\end{array}$ \\
\hline 1967 & $\begin{array}{lll}\mathbf{P}_{\mathrm{I}} & 15 & \mathrm{~h} \\
\mathbf{E}_{\mathrm{I}} & 15 & \mathrm{~h} \\
\mathbf{A}_{\mathrm{I}} & \mathbf{1 5} & \mathrm{h}\end{array}$ & $\begin{array}{l}13 \\
12 \\
13\end{array}$ & $\begin{array}{l}77 \\
42 \\
54\end{array}$ & $\begin{array}{l}5,4 \pm 1,2 \\
4,8 \pm 0,8 \\
5,6 \pm 0,5\end{array}$ & $\begin{array}{l}95 \\
76 \\
77\end{array}$ \\
\hline 1968 & $\begin{array}{lll}\mathrm{P}_{I} & 15 & \mathrm{~h} \\
\mathrm{P}_{I I} & 15 & \mathrm{~h} \\
\mathrm{~A}_{I} & 15 & \mathrm{~h} \\
\mathrm{~A}_{\text {II }} & 15 & \mathrm{~h} \\
\mathrm{P}_{\mathrm{I}} & 10 & \mathrm{~h} \\
\mathrm{P}_{I I} & 10 & \mathrm{~h} \\
\mathrm{~A}_{\mathrm{I}} & 10 & \mathrm{~h} \\
\mathrm{~A}_{\text {II }} & 10 & \mathrm{~h}\end{array}$ & $\begin{array}{r}22 \\
15 \\
12 \\
8 \\
12 \\
11 \\
18 \\
8\end{array}$ & $\begin{array}{r}91 \\
67 \\
83 \\
88 \\
100 \\
73 \\
56 \\
25\end{array}$ & $\begin{array}{l}4,7 \pm 0,3 \\
4,6 \pm 0,6 \\
4,4 \pm 0,8 \\
3,4 \pm 0,6 \\
3,3 \pm 0,4 \\
3,9 \pm 0,5 \\
3,0 \pm 0,3 \\
2,0\end{array}$ & \\
\hline
\end{tabular}

Variations dues aux facteurs

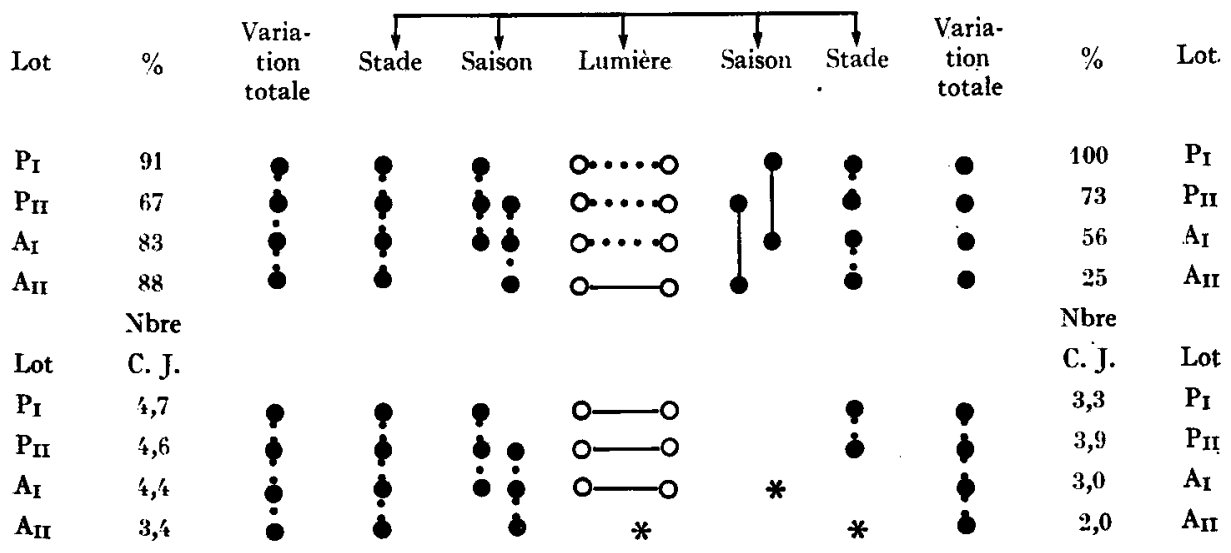

FIG. 4. - Analyse de la variation du nombre de femelles ovulant et du nombre de corps jaunes par femelle ayant ovulé, en fonction des 3 facteurs: stade de développement de la luzerne, saison de la fenaison et durée de la photopériode.

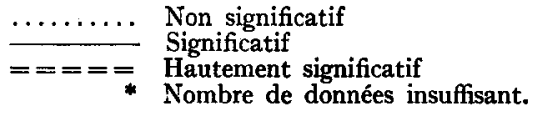


Les luzernes au stade de la préfloraison ont un effet défavorable sur le nombre de femelles ovulant; les différences ne sont pas significatives, sans doute à cause du nombre insuffisant de femelles par lot.

L'effet inhibiteur de luzernes soit d'Automme, soit au stade de la préfloraison, est surtout net pour une photopériode claire de Io heures par 24 heures.

Le nombre de corps jaunes par femelle ne semble modifié que par la durée de la photopériode ; cependant, on peut observer dans les deux lots Automne II, en I968, une diminution du nombre de corps jaunes.

Le pourcentage d'œufs fécondés semble diminuer quand la luzerne est prélevée en Été ou en Automne, plutôt qu'au Printemps. La technique utilisée en I968 n'a pas permis de confirmer ce résultat.

\section{DISCUSSION}

L'ensemble des résultats montre que la mortalité au cours du premier mois de la vie est augmentée, la croissance corporelle et la fertilité des mâles et des femelles diminuées, lorsque les animaux sont nourris :

- avec de la luzerne d'Été ou d'Automne de $30 \mathrm{~cm}$ ou en préfloraison ;

- avec de la luzerne du même cycle végétatif, mais fauchée au stade de la préfloraison par rapport au stade de $30 \mathrm{~cm}$.

Ces effets sont considérablement augmentés lorsque les animaux sont, en plus, soumis à une photopériode claire courte (Io h. par $24 \mathrm{~h}$ ), qui ne favorise pas la croissance et la fertilité comme une photopériode claire longue.

TABLEAU 7

Fertilité des Campagnols en fonction de la saison et du stade de développement de la luzerne

\begin{tabular}{|c|c|c|c|c|}
\hline \multirow{2}{*}{ Année } & \multirow{2}{*}{ Lot } & $\begin{array}{l}\text { Nombre de mâles } \\
\text { fertiles à } 45 \text { jours }\end{array}$ & $\begin{array}{l}\text { Nombre de femelles } \\
\text { fertiles à } 35 \text { jours }\end{array}$ & $\begin{array}{l}\text { Nombre de jeunes } \\
\text { produits }\end{array}$ \\
\hline & & p. 100 campagnols nés & p. 100 campagnols nés & par 100 femelles \\
\hline 1967 & $\begin{array}{lll}\mathrm{P}_{\mathrm{I}} & 15 \mathrm{~h} \\
\mathrm{E}_{\mathrm{I}} & 15 \mathrm{~h} \\
\mathrm{~A}_{\mathrm{I}} & 15 \mathrm{~h} \\
\mathrm{P}_{\mathrm{I}} & 10 \mathrm{~h} \\
\mathrm{E}_{\mathrm{I}} & 10 \mathrm{~h} \\
\mathrm{~A}_{\mathrm{I}} & 10 \mathrm{~h}\end{array}$ & $\begin{array}{l}44 \\
41 \\
30 \\
44 \\
19 \\
15\end{array}$ & $\begin{array}{l}31 \\
17 \\
16 \\
- \\
-\end{array}$ & $\begin{array}{r}184 \\
82 \\
90 \\
- \\
- \\
-\end{array}$ \\
\hline 1968 & $\begin{array}{lll}P_{I} & 15 h \\
P_{I I} & 15 h \\
A_{I} & 15 h \\
A_{I I} & 15 h \\
P_{I} & 10 h \\
P_{I I} & 10 h \\
A_{I} & 10 h \\
A_{I I} & 10 h\end{array}$ & $\begin{array}{l}46 \\
30 \\
35 \\
23 \\
29 \\
2 ! \\
26 \\
16\end{array}$ & $\begin{array}{r}42 \\
21 \\
29 \\
24 \\
31 \\
24 \\
25 \\
5\end{array}$ & $\begin{array}{r}197 \\
97 \\
128 \\
82 \\
102 \\
94 \\
75 \\
10\end{array}$ \\
\hline
\end{tabular}


Dans le tableau 7 , le nombre de mâles ou de femelles fertiles obtenu à partir de roo jeunes nés a été calculé suivant la formule :

roo $\times \frac{\text { Nombre de Jeunes à } 30 \text { jours }}{\text { Nombre de Jeunes nés }} \times \frac{\text { Nombre de } \sigma^{\star} \text { ou } q \text { fertiles }}{\text { Nombre de } \sigma^{\star} \text { ou } q \text { infertiles }}$ et le nombre de jeunes produits par roo femelles de 35 jours, suivant la formule :
roo $x$
Nombre + ayant ovulé
Nombre de corps jaunes chez
Nombre o n'ayant pas ovulé
les femelles ayant ovulé

Lorsque sont associés les trois facteurs : stade de développement de la luzerne, cycle de végétation et durée de la photopériode, il est possible d'expliquer les cycles saisonniers observés dans la nature.

Pinter et Negus (I965, I966, I968) ont montré l'addition des effets dus au photopériodisme et à la qualité de la nourriture sur la croissance et la fertilité de Microtus montanus. D'autre part, de nombreux auteurs, notamment BENDELI（I959), KALEI,A (I96I), Stodart et Mvers (I966) ont essayé d'associer les fluctuations annuelles du nombre des petits Mammifères herbivores avec les changements saisonniers de la qualité des herbages. HoFrmaN (r958) a trouvé un parallélisme au cours de l'année entre la teneur en protéines d'une prairie et le nombre de corps jaunes chez Microtus montanus.

Mais ce ne sont sans doute pas les variations de teneur d'un seul constituant qui sont responsables des effets observés au cours de l'année ; il est fort probable que la quantité de nourriture consommée doit varier aussi en fonction de la teneur en certains constituants, comme la cellulose par exemple.

De toute façon, il semble bien que la présence des deux facteurs végétation et photopériode soit nécessaire pour déterminer le cycle annuel de reproduction chez le Campagnol.

Reçu pour publication en avril 1969.

\section{REMERCIEMENTS}

Nous remercions le Laboratoire d'Amélioration des Plantes fourragères de l'I. N. R. A. qui a mis à notre disposition des parcelles de luzerne et nous a aidés pour l'exécution de l'expérience.

\section{SUMMARY}

EFFECT OF THE SEASONAL CHANGE IN LUCERN FEED ON GROWTH, MORTALITY AND SEXUAL MATURATION OF THE FIELD VOLE " MiCROTUS ARVALIS"

The interaction of plant feeds and day light on growth, mortality and sexual maturation were studied in the field vole (Microtus arvalis).

The animals were kept from birth under Io or $I_{5}$ hour periods of daily light and fed with lucern hay cut in Spring or Autumn at two different stages ( $30 \mathrm{~cm}$ of preflorescence).

First month mortality was greater, body growth and male or female fertility lower, in batches submitted to Io hours of daily lighting and fed lucern hay cut in Autoumn in its preflorescent stage.

The natural seasonal cycles observed in populations of voles can be tentatively accounted for by the cumulative effects of the three factors, plant growth stage, season of cut, and photoperiod. 


\section{RÉFÉRENCES BIBLIOGRAPHIQUES}

Bendell J. F., r 959. Food as a control of a population of white footed mice, Peromyscus Leucopus. Can. J. Zool., 37, $173^{-209}$.

Bernard J., I 964 . Note préliminaire sur la reproduction chez le Campagnol des Champs Microlus arvalis dans le sud de la Belgique. Bull. Inst. agron. Sta. Rech. Gembloux, 82, 275-289.

Delost P., 1955. Etude de la biologie sexuelle du Campagnol des Champs (Microtus arvalis). Arch. Anat. micr. Morph. exp., 44, 1 50-190.

Dounce A. L., I 943. Further studies on isolated cell nuclei of normal rat liver. J. Biol. Chem., 151, 22 :-233.

HomfFan R.S., I $95^{8}$. The role of reproduction and mortality in population fluctuations of voles (Microtus). Ecolog. Monog., 28, 79-09.

KalELA O., 196I. Seasonal change of habitat in the Norvegian Lemming (Lemmus Lemmus). Acad. Scient. Fenn. Biologica, 55, 1-72.

LECYK M., r 1962. The effect of the length of day light on reproduction in the field vole (Microtus arvalis). Zool. Pol., 178, i 89-22r.

ManN T., 1946. Studies on the metabolism of semen. III. Fructose as a normal constituant of seminal plasma. Site of formation and function of fructose in semen. Biochem. J., 40, 48:-49:.

Martinet L., I966. Modification de la spermatogenèse chez le Campagnol des Champs (Microtus arvalis) en fonction de la durée quotidienne d'éclairement. Ann. Biol. anim. Bioch. Biophys., 6, 3०1-3 3.

Martiner L., r967. Cycle saisonnier de reproduction du Campagnol des Champs Microtus arvalis. Ann. Biol. anim. Bioch. Biophys., 7, 245-260.

Negus N. C., Pinter A. J., I 966 . Reproductive responses of Microtus montanus to plants and plants extracts in the diet. J. Mammal., 47, 596-60r.

Pinter A. J., Negus N. C., I 965 . Effects of nutrition and photoperiod on reproductive physiology of Microlus montanus. Amer. J. physiol., 208, 633-641.

Pinter A. J, ] 968 . Effects of diet and light on growth maturation and adrenal size of Microlus montanus. Amer. J. Physiol., 215, 46:-466.

STEı N H. G. W., I953. Uber das Zalhenverhältniss der geschlechter bei der Feldmaus, Microlus arvalis. Zool. Jahrb. Abt. Syst,, 82, $137^{-i} 5^{6 .}$

Stodart F., Myers K., I 966 . The effects of different foods on confined populations of wild rabbits Oryctolagus cuniculus. C.S. T. R. O. Wildl Res., 11, I I I-1 24 . 